\title{
Research Article \\ Sensitivity Analysis for the Mechanical Properties of DNA Bundles
}

\author{
Young-Joo Kim ${ }^{1}$ and Do-Nyun Kim ${ }^{1,2}$ \\ ${ }^{1}$ Department of Mechanical and Aerospace Engineering, Seoul National University, Building 301, Room 1516, Gwanak-ro 1, \\ Gwanak-gu, Seoul 08826, Republic of Korea \\ ${ }^{2}$ Institute of Advanced Machines and Design, Seoul National University, Gwanak-ro 1, Gwanak-gu, Seoul 08826, Republic of Korea \\ Correspondence should be addressed to Do-Nyun Kim; dnkim@snu.ac.kr
}

Received 31 December 2015; Accepted 19 April 2016

Academic Editor: Serdal Kirmizialtin

Copyright (c) 2016 Y.-J. Kim and D.-N. Kim. This is an open access article distributed under the Creative Commons Attribution License, which permits unrestricted use, distribution, and reproduction in any medium, provided the original work is properly cited.

In structural DNA nanotechnology, programming a three-dimensional shape into DNA bundles has been a primary design objective. However, the mechanical properties of these DNA bundle structures are another important factor to be considered in the design process. While the mechanics of the individual DNA double helix has been explored extensively and hence its properties are well known, the mechanical properties of structural motifs such as DNA junctions and strand breaks important to bundle mechanics have not been well characterized due to experimental limitations, rendering it difficult to predict the mechanical properties of DNA bundles. Here, we investigate the effect of these structural motifs on the global bundle rigidities by performing sensitivity analysis on a six-helix DNA bundle structure using the finite element modeling approach. Results reveal the primary structural features and their parametric values required to reproduce the experimental bundle rigidities.

\section{Introduction}

Structural DNA nanotechnology has enabled us to build various molecular structures with desired patterns and shapes at the nanometer scale $[1,2]$. By designing a connectivity map between DNA double helices at discrete crossover positions, one can fold DNA strands into the two- to three-dimensional structures [2-8] with target curvatures or twists $[9,10]$. These structures have been widely used in many applications including the nanometer-precision arrangement of nanoparticles, proteins or florescence molecules [11-13], the controlled growth of gold nanoparticles into a three-dimensional shape by using DNA nanostructures as a mold $[14,15]$, and the measurement of single-molecule properties [16].

While the solution shape has been a primary design objective for constructing a target DNA nanostructure, the mechanical properties should be also taken into consideration in the design process as they not only govern the stability, flexibility, and deformability of the structure but also affect the derived functional properties facilitated by interacting with molecules or particles attached to it. Although studies on the mechanical properties of the B-form DNA double helix are abundant, those for DNA bundles [17-19] are scarce because of the lack of information on the mechanical properties of structural motifs such as DNA junctions and strand breaks important to bundle mechanics due to experimental limitation. Therefore, it is difficult to predict the mechanical properties of DNA bundles in the design process.

Here, we investigate the effect of the mechanical properties of structural motifs, which have been ill-characterized and largely unknown, on the DNA bundle rigidities by analyzing a six-helix DNA bundle structure whose bending and torsional rigidities were known experimentally [17]. We employ the finite element (FE) method to calculate the bundle rigidities for systematically varied model parameters including the distance between the adjacent helices and the rigidities of crossovers and nicks. Results reveal plausible roles of these parameters on the global bundle rigidities and provide their values reproducing the experimental bundle properties. 
(a)

(b)

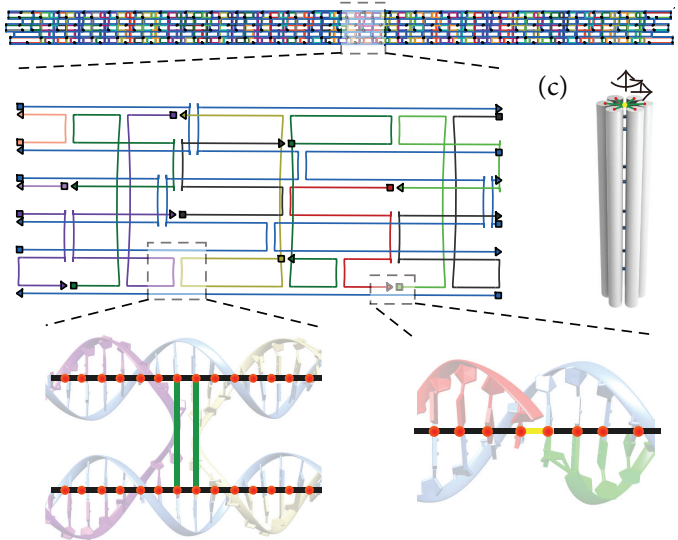

FIgURE 1: Six-helix DNA bundle structure. (a-b) Two-dimensional blueprint, (c) three-dimensional rendering of the structure, and (d) finite element models of a crossover (left) and a nick (right). In (d), black, green, and yellow lines represent beam elements used to model normal DNA double helices, crossovers, and nicks, respectively. Red dots are FE nodes.

\section{Materials and Methods}

2.1. Six-Helix Bundle. The six-helix DNA bundle [17] is a straight, $371 \mathrm{~nm}$ long structure designed in the honeycomb lattice and consisting of a 6552-base-long scaffold strand and 156 short staple strands most of which have 42 bases (Figure 1). Originally, it has two supporting parts at both ends of the structure for experiments, but we excluded them in this study as they are not necessary in our computational analysis. The helices are connected discretely at 595 crossover positions that are almost evenly distributed along the helical axis. 156 strand breaks or nicks exist almost every 42 base pairs in the bundle as it is folded by the short staple strands. More details on the six-helix DNA bundle are available in [17] including sequence information of comprising DNA strands, connectivity maps between helices, and experimental methods to synthesize and characterize the bundle.

2.2. Finite Element Model. The three-dimensional FE model of the six-helix DNA bundle structure is constructed from its two-dimensional blueprint [20] that provides the location of each helix on a lattice and the crossover positions where the helices are connected to one another. Each helix is modeled as a straight, homogeneous, isotropic, and elastic rod using a set of two-node Hermitian beam elements with the stretching $\left(S_{\mathrm{B}-\mathrm{DNA}}=1,100 \mathrm{pN}\right)$, bending $\left(B_{\mathrm{B}-\mathrm{DNA}}=230 \mathrm{pNnm}^{2}\right)$, and torsional $\left(C_{\mathrm{B}-\mathrm{DNA}}=460 \mathrm{pNnm}^{2}\right)$ rigidities measured experimentally for the B-form DNA [21] neglecting any sequence dependency. FE nodes are placed at the center of base pairs where six degrees of freedom (DOFs) are assigned to each node to describe the displacement of the center position and the rotation of the beam cross section. They exist every $0.34 \mathrm{~nm}$ along the helical axis in the undeformed configuration with the right-handed twist rate of $34.29^{\circ}$ per base pair determining the orientation of the beam cross section. At the nick positions where one of the phosphate backbones is broken, beams with reduced bending and torsional rigidities are used instead while the same stretching rigidity as the B-form DNA is used. Six helices form a bundle structure on the honeycomb lattice with the interhelical distance between the neighboring helices. Two FE nodes between the adjacent helices at a crossover position are connected to each other using a beam element whose rigidities determine the flexibilities between the helices at the junction position.

2.3. Sensitivity Analysis. We investigate the sensitivity of the bundle rigidities on the principal structural parameters, which are difficult to measure experimentally, governing the geometry and the mechanical properties of the six-helix DNA bundle structure. We calculate the stretching $(S)$, bending $(B)$, and torsional $(C)$ rigidities of the bundle for exhaustively varied parametric values including the interhelical distance $(D)$, the reduced bending $\left(B_{\text {nick }}\right)$ and torsional $\left(C_{\text {nick }}\right)$ rigidities of the beam element at nicks, and the stretching $\left(S_{\text {cross }}\right)$, bending $\left(B_{\text {cross }}\right)$, and torsional $\left(C_{\text {cross }}\right)$ rigidities of the beam element at crossover positions. At the ends of the bundle, two fictitious FE nodes are generated on the mean position of the helix centers and connected rigidly to the nodes on the same cross-sectional plane where boundary conditions and loading conditions are applied. $S, B$, and $C$ of the bundle for each parameter set are obtained by performing linear static analysis where we impose a unit tensile displacement, a unit shear displacement for bending, or a unit torsional angle on one end of the bundle while fixing the other end and measure the axial reaction force, the shear reaction force, or the torsional reaction moment, respectively. The default values used for these parameters when not varied are $D=$ $2.25 \mathrm{~nm}, S_{\text {cross }} / S_{\text {B-DNA }}=B_{\text {cross }} / B_{\text {B-DNA }}=C_{\text {cross }} / C_{\text {B-DNA }}=$ $10^{5}$, and $B_{\text {nick }} / B_{\mathrm{B}-\mathrm{DNA}}=C_{\text {nick }} / C_{\mathrm{B}-\mathrm{DNA}}=10^{-2}$.

\section{Results and Discussion}

3.1. Isotropic Bending Rigidity. Due to the helicity of the B-form DNA (two full turns per 21 base pairs), crossover positions are rotating 240 degrees every seven base pairs along the helical axis on the honeycomb lattice, resulting in an asymmetric crosslinking arrangement. Therefore, the bending rigidity of the bundle naturally varies with the direction of curvature. The bundle exhibits the highest bending rigidity for bending along the vertical axis while it shows the lowest rigidity for bending along the horizontal axis. Nevertheless, the difference between the maximum and minimum bending rigidities is negligibly small (less than $0.2 \%$ of the mean value) and hence we can assume that the bending rigidity of the bundle is almost isotropic (Figure 2). This is because the bundle uses the highest crossover density available for honeycomb-latticed structures. More specifically, a DNA double helix in the six-helix bundle is connected to one of its three neighboring helices every seven base pairs forming a 21base-pair-long repeating unit. Approximately 50 units exist along the helical axis that are large enough to attenuate the effect of crossover asymmetry on the overall bending rigidity of the bundle. 


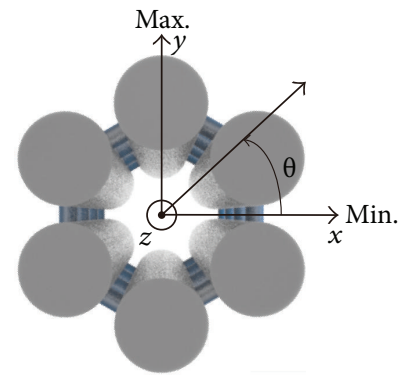

(a)

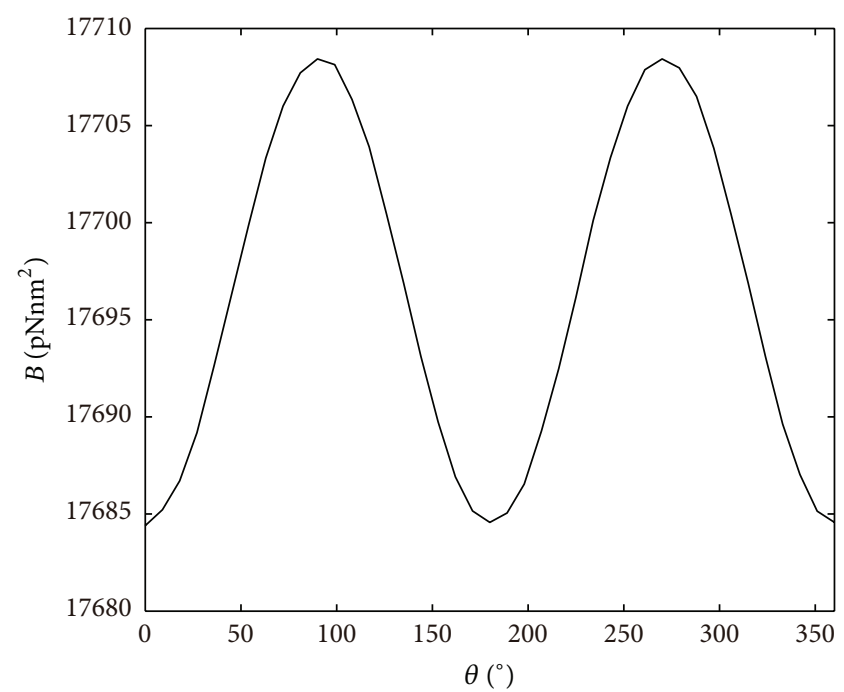

(b)

FIGURE 2: Bending rigidity. (a) Definition of the axis along which the bundle bends and (b) the bending rigidity of the bundle.

3.2. Effect of the Interhelical Distance. Here, we investigate the effect of the distance between the helices on the overall bundle rigidities. It is well known that the diameter of the Bform DNA is about $2 \mathrm{~nm}$, which might be a natural choice for the interhelical distance if we assume that the helices are in contact with one another almost all the way along the helical axis. However, as the DNA double helix is highly negatively charged, the repulsive forces whose strength is dependent on the ionic condition exist between the helices. On the other hand, it is shown experimentally that the interhelical distance at crossover positions is smaller than the helix diameter amounting to $1.85 \mathrm{~nm}$. As a result, the helices undulate in the bundle rather than taking a straight, parallel configuration because they expel one another while constrained at crossover positions. Nonetheless, we often model the structure as a bundle of straight DNA helices separated at an effective interhelical distance corresponding to the mean distance between the undulating helices without including electrostatics explicitly into the model, which has been successful to predict the three-dimensional shape of DNA origami nanostructures $[1,22]$.

Here, we adopt this modeling approach and study how the effective interhelical distance affects the bundle rigidities by varying it from $1.8 \mathrm{~nm}$ to $2.5 \mathrm{~nm}$. Results clearly demonstrate that the stretching rigidity of the bundle is invariant to the interhelical distance (Figure 3(a)). This is because the axial force is divided and applied equally to each helix resulting in a uniform axial deformation of the helices regardless of their relative locations. Bending and torsional rigidities of the bundle, in contrast, are dependent on the interhelical distance (Figures 3(b) and 3(c)) resulting primarily from the fact that area moments of inertia increase with the interhelical distance as the helices become located farther from the bundle's central axis.
3.3. Effect of the Mechanical Properties of Crossovers. The mechanical properties of crossovers are the most crucial factors affecting the global bundle rigidities as DNA double helices are interconnected only at these discrete positions. These crossovers are four-way Holliday junctions that are structurally polymorphic when free in solution and can undergo the transition between an unstable, open conformer and more stable, stacked conformers [23]. These junction structures take a right-handed stacked conformation in a bundle under usual ionic conditions and provide the crosslinking stiffness between the adjacent helices. While it has been shown both experimentally and computationally that crossovers are quite flexible in scissor-like motion of junction arms [5, 24], their exact mechanical properties have not been characterized well. Here we explore how the mechanical properties of crossovers affect the bending and torsional rigidities of the six-helix bundle by varying the stretching, bending, and torsional rigidities of crossovers. The stretching rigidity of the bundle does not change with the crossover rigidities as all six helices are stretched or compressed uniformly.

Results indicate that both bending and torsional rigidities of the bundle are almost independent of the stretching rigidity of crossovers (Figure 4). This is because the global bending or twisting of the bundle requires the bending or twisting of individual helices relative to one another without the change in the interhelical distance. On the contrary, the bending rigidity of crossovers exerts a strong influence on the bundle rigidity in both bending and torsion. As crossovers become flexible in bending, beam elements across the neighboring helices at crossover positions can bend under externally applied bending or torsional moments, resulting in the decrease of the interhelical distance and the consequent reduction of the bundle rigidities (Figure 3). The torsional rigidity of crossovers controlling the scissor-like rotation 


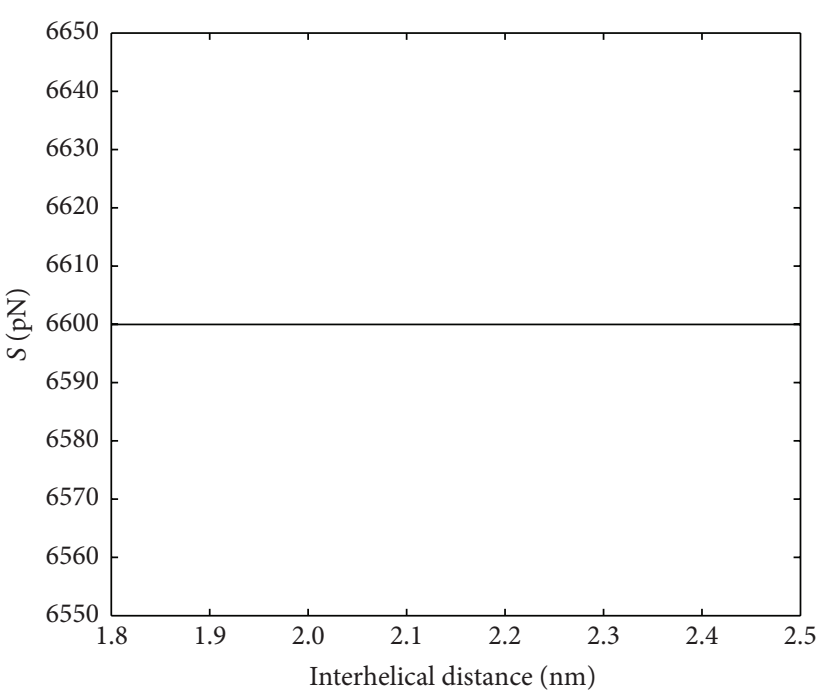

(a)

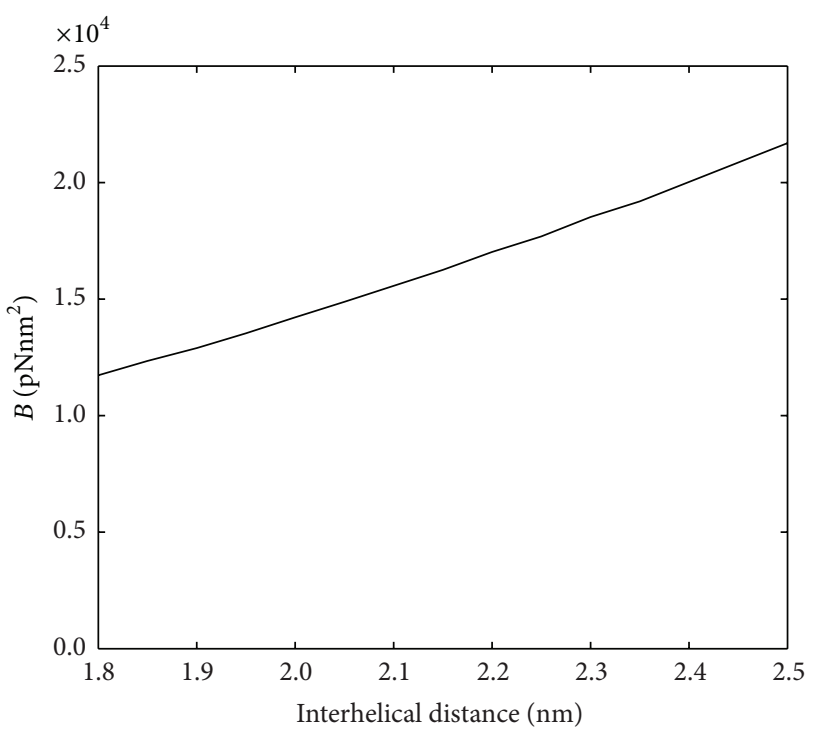

(b)

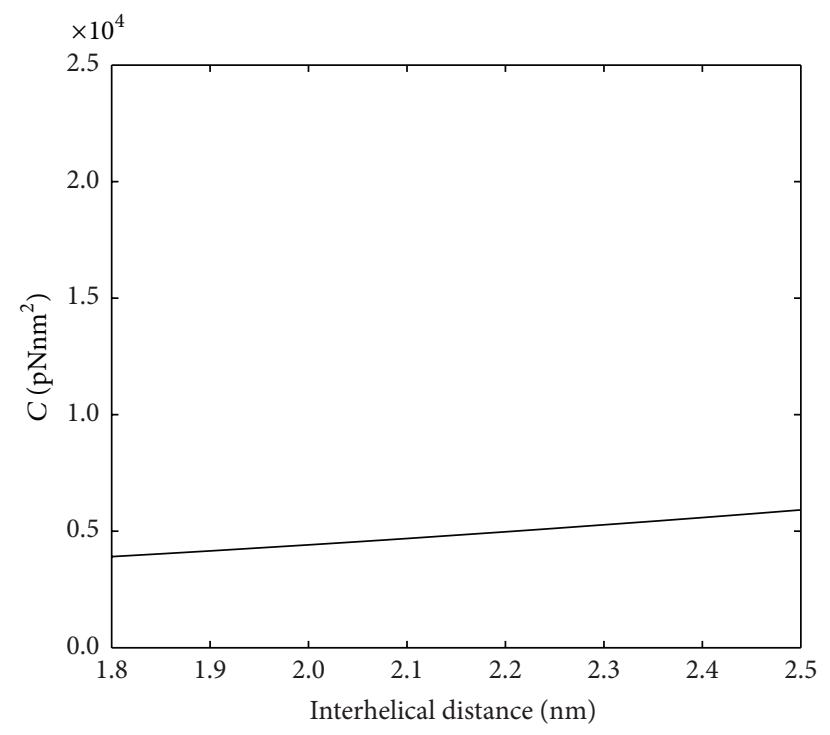

(c)

FIGURE 3: Effect of the interhelical distance. (a) Stretching, (b) bending, and (c) torsional rigidities of the bundle at various interhelical distances.

of the interconnected helices affects the torsional bundle rigidity only since the scissor-like rotation does not occur in bending of the bundle. It is noteworthy that both bending and torsional rigidities of crossovers should be smaller than those of the B-form DNA double helix in order to reproduce the experimental bundle rigidities.

3.4. Effect of the Mechanical Properties of Nicks. As DNA bundles are formed by the aid of many short single stranded DNA segments, nicks (or strand breaks) always exist in a DNA bundle and hence it is important to understand their mechanical roles on the bundle rigidities. However, the mechanical properties of nicks have not been well quantified yet while there is a general consensus that they act like a hinge or swivel with smaller rigidities than those of nonnicked DNA. Hence, we explore the effect of the mechanical properties of nicks on the bundle rigidities by varying the bending and torsional rigidities of nicks while fixing their stretching rigidity to that of the $\mathrm{B}$-form DNA.

It turns out that nicks affect the torsional rigidity of the bundle but have a negligible influence on the bending rigidity (Figure 5). In torsion, the bundle is more sensitive to the torsional rigidity of nicks than the bending rigidity as individual helices can be twisted locally at nick positions in addition to global twist of the bundle. But, if the bending rigidity of nicks is sufficiently small, then it can reduce the torsional rigidity of the bundle significantly as individual helices now can be bent or kinked locally at nick positions under torsion. Therefore, we need to take the location, the density, and the mechanical 


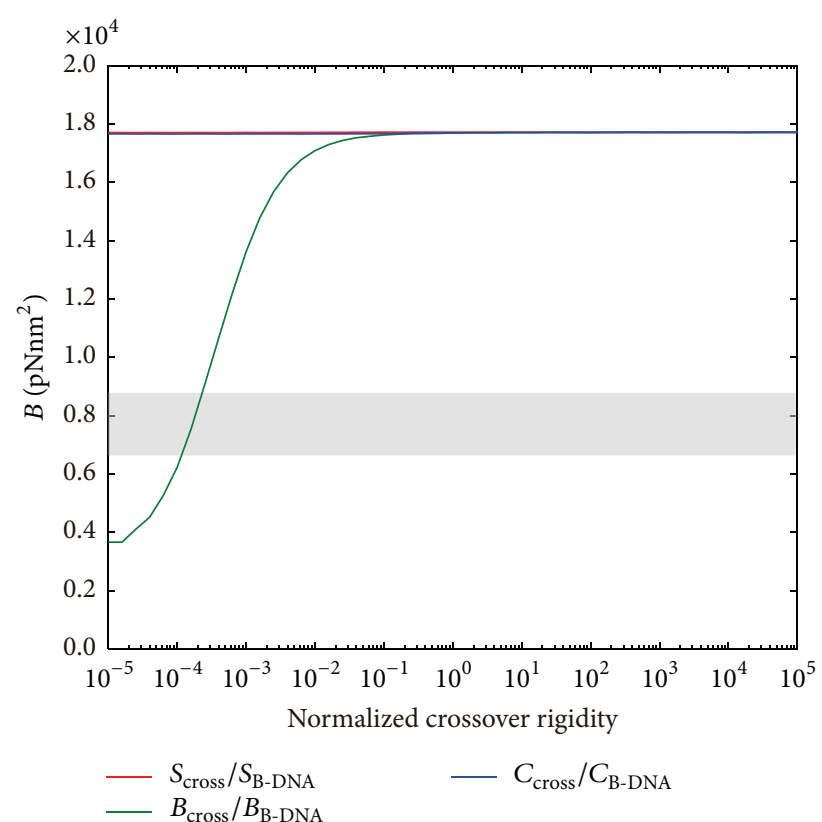

(a)

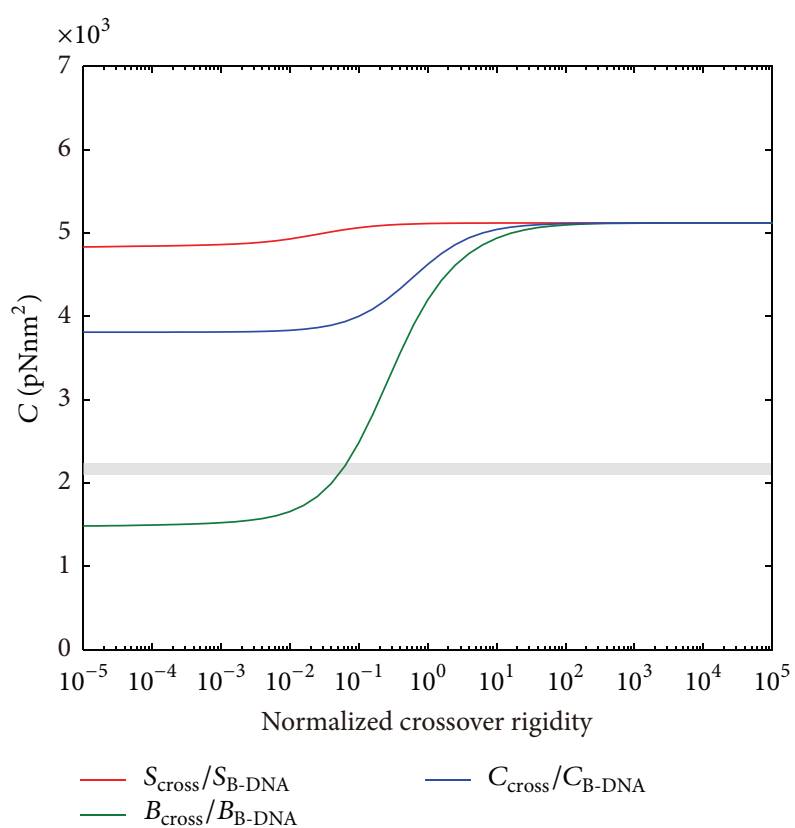

(b)

FIGURE 4: Effect of the mechanical properties of crossovers. (a) Bending and (b) torsional rigidities of the bundle computed using various crossover rigidities. Shaded areas represent the experimentally measured rigidities.

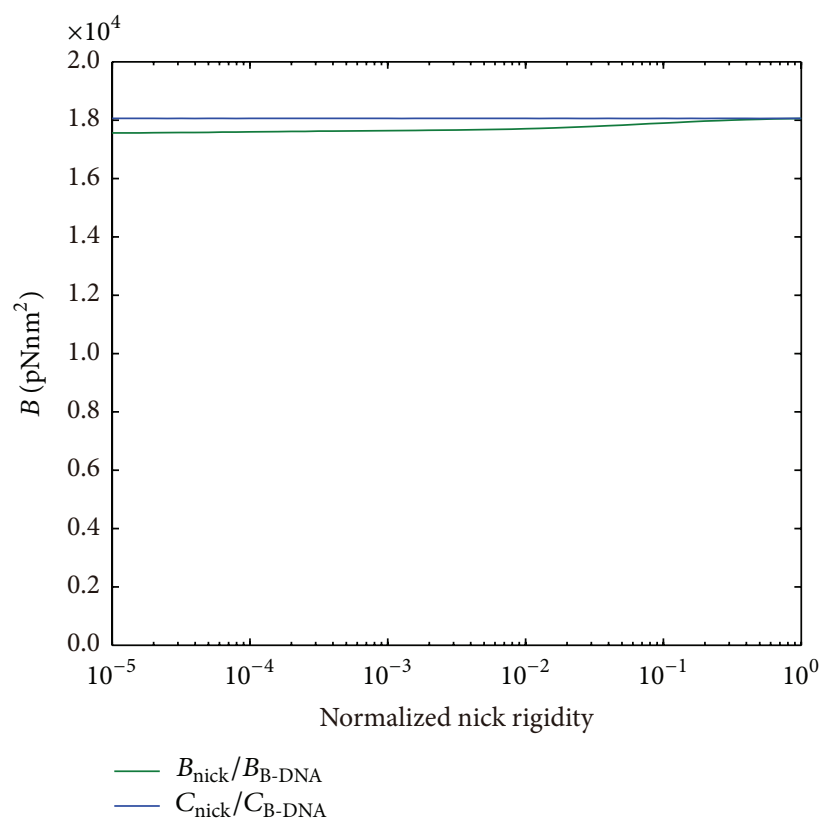

(a)

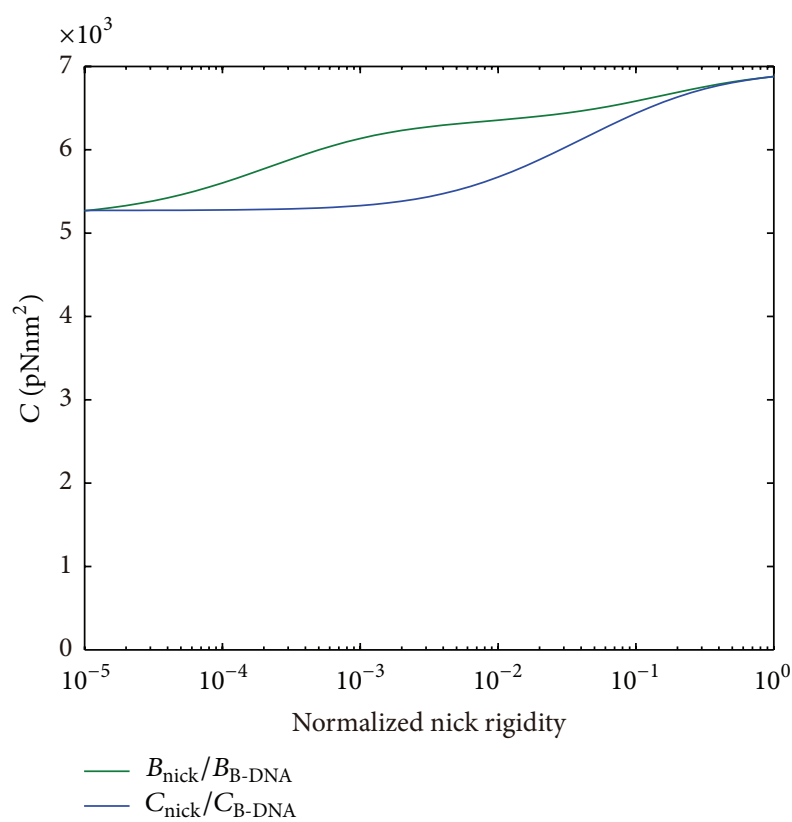

(b)

FIGURE 5: Effect of the mechanical properties of nicks. (a) Bending and (b) torsional rigidities of the bundle computed using various nick rigidities.

properties of nicks into consideration when we design a DNA bundle structure exposed to torsional loadings.

3.5. Comparison with Other Models. Based on the results of sensitivity analysis, we can estimate the parametric values of the model reproducing the bending and torsional rigidities of the six-helix bundle measured experimentally. If we use the interhelical distance of $2.25 \mathrm{~nm}$ representing an effective helical diameter at usual ionic concentrations used for DNA bundles, the model predicts the bending and torsional rigidities of $7,247 \mathrm{pNnm}^{2}$ and 2,198 $\mathrm{pNnm}^{2}$, respectively, for the bundle. These values lie in the range of experimental rigidities, 6,624 $8,846 \mathrm{pNnm}^{2}$ for bending and 2,098 2,263 $\mathrm{pNnm}^{2}$ for torsion corresponding to one standard deviation from the mean 


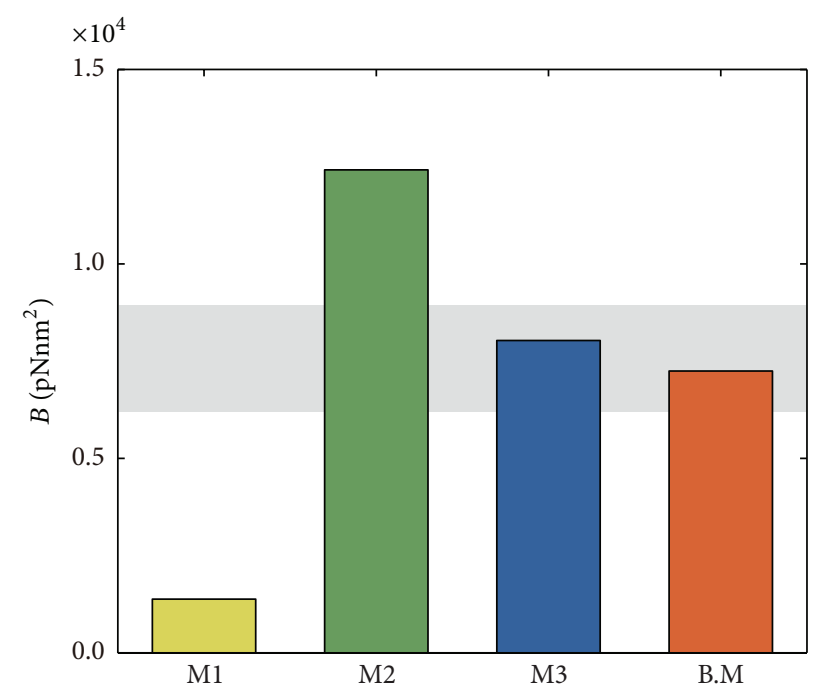

(a)

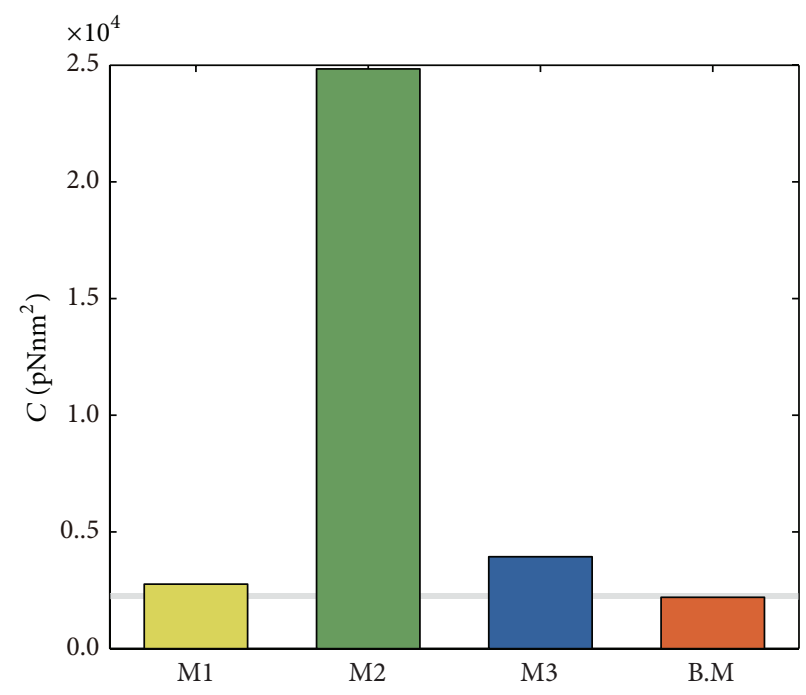

(b)

FIGURE 6: Bundle rigidities computed using several DNA models. (a) Bending and (b) torsional rigidities of the bundle. M1, M2, and M3 are continuum models where each DNA double helix is modeled as an isotropic cylinder. The helices are not connected in M1, continuously connected over the helices in M2, and discretely connected only at crossover positions in M3. B.M represents the beam model of the bundle (Figure 1). Shaded areas represent the experimentally measured rigidities.

rigidities [17]. Parametric values used to obtain these bundle rigidities are $B_{\text {cross }} / B_{\mathrm{B}-\mathrm{DNA}}=0.000125, C_{\text {cross }} / C_{\mathrm{B}-\mathrm{DNA}}=0.1$, and $B_{\text {nick }} / B_{\mathrm{B}-\mathrm{DNA}}=C_{\text {nick }} / C_{\mathrm{B} \text {-DNA }}=0.065$ indicating that both crossovers and nicks are much softer than the B-form DNA. The torsional rigidity of crossovers inferred from our sensitivity analysis is similar to the one obtained using the molecular dynamics simulation [24]. It would be valuable to investigate the other mechanical properties of crossovers and nicks using atomistic simulations as well in order to further validate our analysis and obtain molecular-level insights into their properties.

When compared with other models, the capabilities of the beam finite element model become more prominent (Figure 6). Three continuum bundle models [17] are considered where each DNA double helix is treated as an isotropic cylinder characterized by its Young's modulus and Poisson's ratio. These models are distinguished by the assumption in crosslinking the helices: no crosslink (M1), continuous crosslink over the entire helix (M2), and discrete crosslink at crossover positions (M3). M2 predicts excessively high rigidities of the bundle particularly in torsion while M1 predicts too low rigidities particularly in bending. M3 predicts the bundle rigidities close to experimental ones, but it is noteworthy that two different Young's moduli had to be used for bending and torsion because M3 is unable to reproduce the bending and torsional rigidities of the bundle simultaneously using single Young's modulus.

\section{Conclusions}

In this paper, we investigate the mechanical properties of DNA bundles by performing sensitivity analysis for the six-helix DNA bundle structure using the finite element modeling approach. The bending and torsional rigidities of the bundle that are measured experimentally are calculated using our computational model with systematically varied parametric values including the interhelical distance, the crossover rigidities, and the nick rigidities. Results reveal that (1) the bending rigidity of the bundle is almost isotropic as a sufficiently large number of crossovers are used, (2) the interhelical distance affects the bending and torsional rigidities but has no influence on the stretching rigidity of the bundle, (3) the bending rigidity of crossovers has a dominant effect on the bundle rigidities, and (4) nicks are important for the torsional rigidity of the bundle. In addition, it is shown that the beam model can reproduce the experimental bundle rigidities while the previous continuum models cannot. Our findings are expected to be useful to design more complex DNA-based nanostructures with target shapes, mechanical properties, and derived functional properties.

\section{Competing Interests}

The authors declare that they have no competing interests.

\section{Acknowledgments}

This research was supported by the Basic Science Research Program (Grant no. 2013R1A1A1010626) through the National Research Foundation of Korea (NRF) and by the Center for Advanced Meta-Materials (CAMM) as Global Frontier Project (CAMM-2014M3A6B3063711) funded by the Ministry of Science, ICT and Future Planning.

\section{References}

[1] C. E. Castro, F. Kilchherr, D.-N. Kim et al., "A primer to scaffolded DNA origami," Nature Methods, vol. 8, no. 3, pp. 221-229, 2011. 
[2] P. W. K. Rothemund, "Folding DNA to create nanoscale shapes and patterns," Nature, vol. 440, no. 7082, pp. 297-302, 2006.

[3] B. Wei, M. Dai, and P. Yin, "Complex shapes self-assembled from single-stranded DNA tiles," Nature, vol. 485, no. 7400, pp. 623-626, 2012.

[4] S. M. Douglas, H. Dietz, T. Liedl, B. Högberg, F. Graf, and W. M. Shih, "Self-assembly of DNA into nanoscale three-dimensional shapes," Nature, vol. 459, pp. 414-418, 2009.

[5] D. Han, S. Pal, Y. Yang et al., "DNA gridiron nanostructures based on four-arm junctions," Science, vol. 339, no. 6126, pp. 1412-1415, 2013.

[6] R. Iinuma, Y. Ke, R. Jungmann, T. Schlichthaerle, J. B. Woehrstein, and P. Yin, "Polyhedra self-assembled from DNA tripods and characterized with 3D DNA-PAINT," Science, vol. 344, no. 6179, pp. 65-69, 2014.

[7] Y. Ke, L. L. Ong, W. M. Shih, and P. Yin, "Three-dimensional structures self-assembled from DNA bricks," Science, vol. 338, no. 6111, pp. 1177-1183, 2012.

[8] E. Benson, A. Mohammed, J. Gardell et al., "DNA rendering of polyhedral meshes at the nanoscale," Nature, vol. 523, no. 7561, pp. 441-444, 2015.

[9] H. Dietz, S. M. Douglas, and W. M. Shih, "Folding DNA into twisted and curved nanoscale shapes," Science, vol. 325, no. 5941, pp. 725-730, 2009.

[10] D. Han, S. Pal, J. Nangreave, Z. Deng, Y. Liu, and H. Yan, "DNA origami with complex curvatures in three-dimensional space," Science, vol. 332, no. 6027, pp. 342-346, 2011.

[11] R. Schreiber, N. Luong, Z. Fan et al., "Chiral plasmonic DNA nanostructures with switchable circular dichroism," Nature Communications, vol. 4, article 2948, 2013.

[12] S. Rinker, Y. Ke, Y. Liu, R. Chhabra, and H. Yan, "Self-assembled DNA nanostructures for distance-dependent multivalent ligand-protein binding," Nature Nanotechnology, vol. 3, no. 7, pp. 418-422, 2008

[13] K. Pan, E. Boulais, L. Yang, and M. Bathe, "Structure-based model for light-harvesting properties of nucleic acid nanostructures," Nucleic Acids Research, vol. 42, no. 4, pp. 2159-2170, 2014.

[14] W. Sun, E. Boulais, Y. Hakobyan et al., "Casting inorganic structures with DNA molds," Science, vol. 346, no. 6210, Article ID 1258361, 2014.

[15] S. Helmi, C. Ziegler, D. J. Kauert, and R. Seidel, "Shape-controlled synthesis of gold nanostructures using DNA origami molds," Nano Letters, vol. 14, no. 11, pp. 6693-6698, 2014.

[16] E. Pfitzner, C. Wachauf, F. Kilchherr et al., "Rigid DNA beams for high-resolution single-molecule mechanics," Angewandte Chemie, vol. 125, no. 30, pp. 7920-7925, 2013.

[17] D. J. Kauert, T. Kurth, T. Liedl, and R. Seidel, "Direct mechanical measurements reveal the material properties of threedimensional DNA origami," Nano Letters, vol. 11, no. 12, pp. 5558-5563, 2011.

[18] D. Schiffels, T. Liedl, and D. K. Fygenson, "Nanoscale structure and microscale stiffness of DNA nanotubes," ACS Nano, vol. 7, no. 8, pp. 6700-6710, 2013.

[19] C. E. Castro, H.-J. Su, A. E. Marras, L. Zhou, and J. Johnson, "Mechanical design of DNA nanostructures," Nanoscale, vol. 7, no. 14, pp. 5913-5921, 2015.

[20] S. M. Douglas, A. H. Marblestone, S. Teerapittayanon, A. Vazquez, G. M. Church, and W. M. Shih, "Rapid prototyping of 3D DNA-origami shapes with caDNAno," Nucleic Acids Research, vol. 37, no. 15, pp. 5001-5006, 2009.
[21] J. Gore, Z. Bryant, M. Nöllmann, M. U. Le, N. R. Cozzarelli, and C. Bustamante, "DNA overwinds when stretched," Nature, vol. 442, no. 7104, pp. 836-839, 2006.

[22] D.-N. Kim, F. Kilchherr, H. Dietz, and M. Bathe, "Quantitative prediction of 3D solution shape and flexibility of nucleic acid nanostructures," Nucleic Acids Research, vol. 40, no. 7, pp. 28622868, 2012.

[23] S. A. McKinney, A.-C. Déclais, D. M. J. Lilley, and T. Ha, "Structural dynamics of individual Holliday junctions," Nature Structural Biology, vol. 10, no. 2, pp. 93-97, 2003.

[24] K. Pan, D.-N. Kim, F. Zhang, M. R. Adendorff, H. Yan, and M. Bathe, "Lattice-free prediction of three-dimensional structure of programmed DNA assemblies," Nature Communications, vol. 5, article 5578, 2014. 

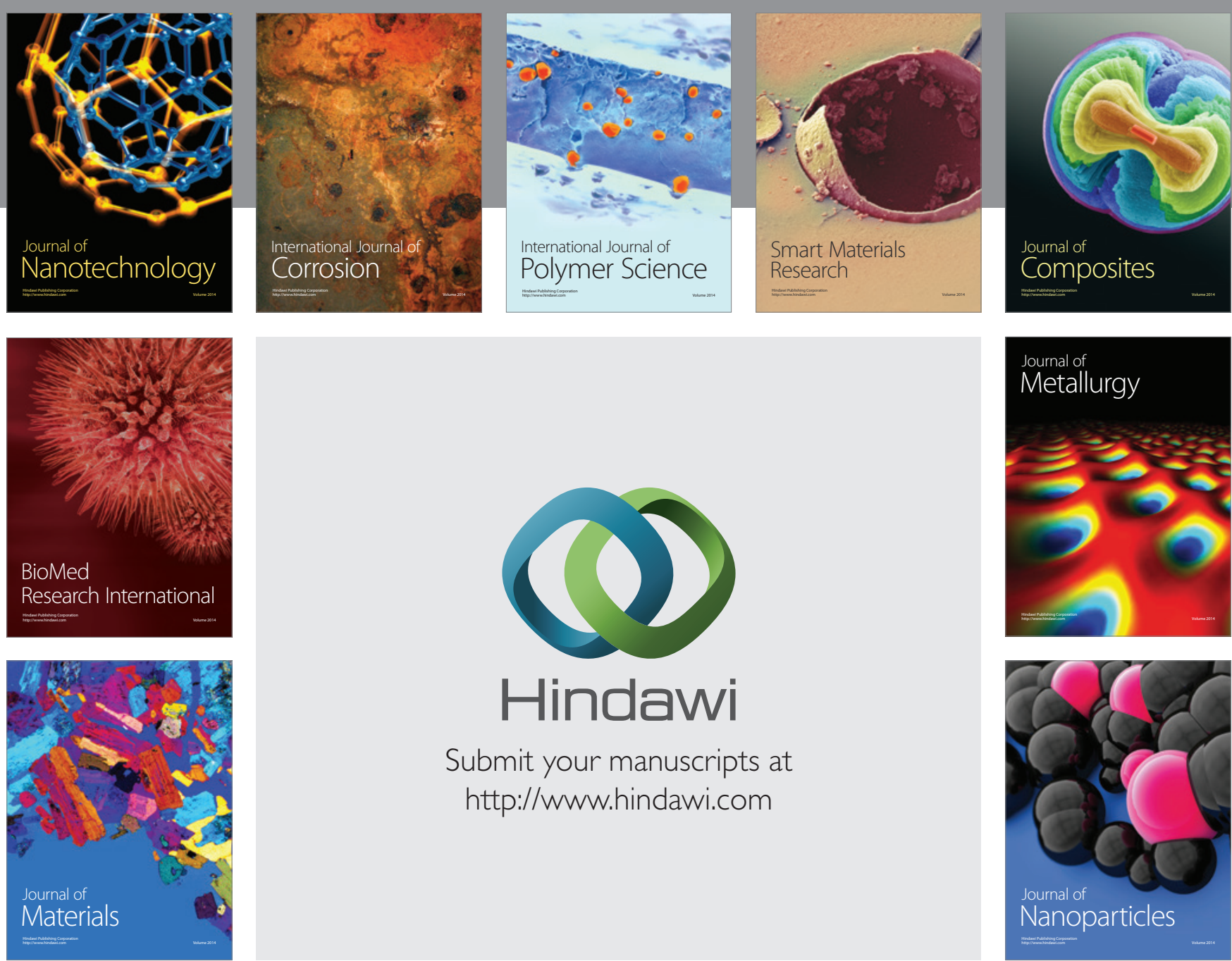

\section{Hindawi}

Submit your manuscripts at

http://www.hindawi.com

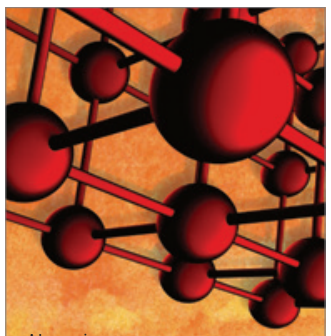

Materials Science and Engineering
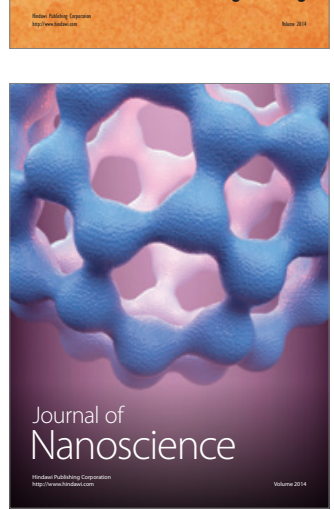
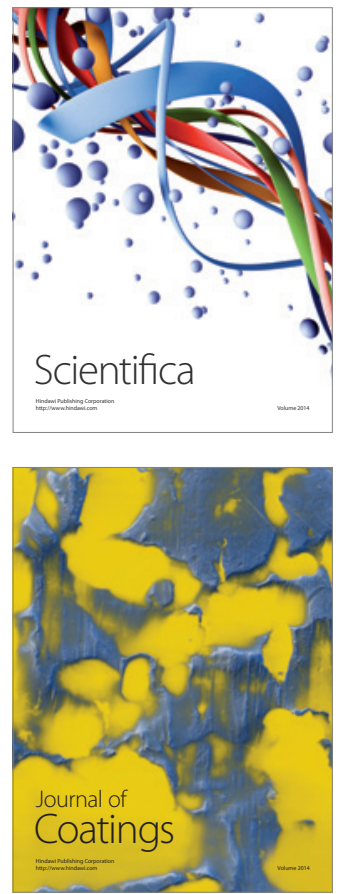
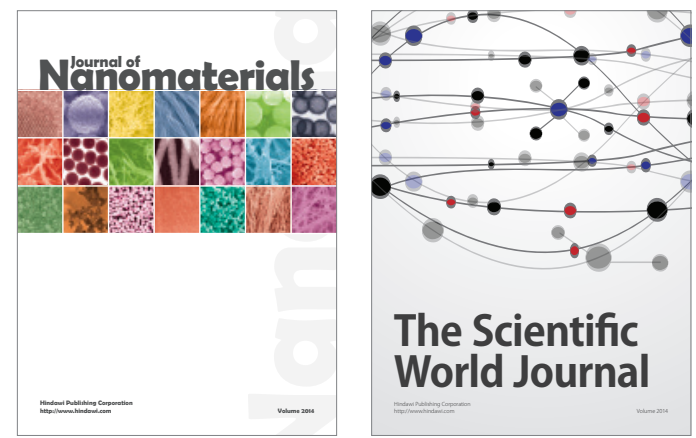

The Scientific World Journal
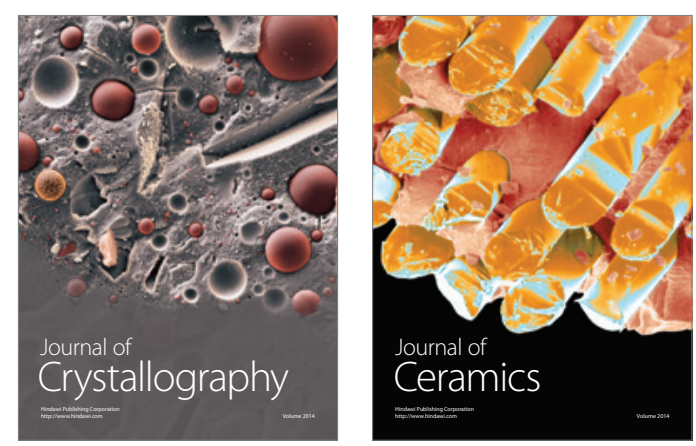
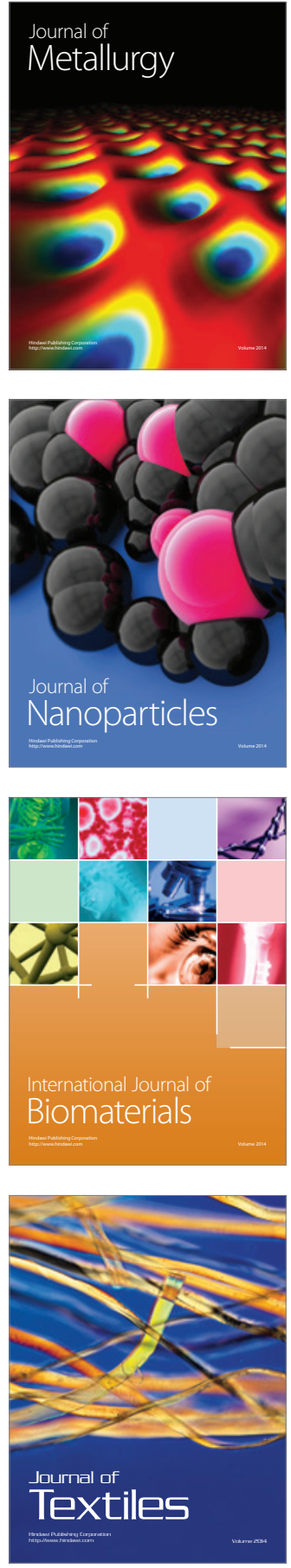